\title{
A case of renal tuberculosis diagnosed during percutaneous nephrolithotomy
}

\author{
Hakan Öztürk ${ }^{1^{*}}$, Musa Saraçoglu ${ }^{1}$, Serap Karaarslan², Tarik Zengin ${ }^{1}$, Aşkın Gülşen ${ }^{3}$, \\ Sena Kalaycıoğlu ${ }^{4}$ \\ ${ }^{1}$ Urology Department, Sifa University, Izmir, Turkey; \\ *Corresponding Author: drhakanozturk@yahoo.com.tr, musasaracoglu@yahoo.com, t.zengin@yahoo.com \\ ${ }^{2}$ Pathology Department, Sifa University, Izmir, Turkey; serapkaraarslan@gmail.com \\ ${ }^{3}$ Chest Diseases Department, Sifa University, Izmir, Turkey; askingulsen@hotmail.com \\ ${ }^{4}$ Radiology Department, Sifa University, Izmir, Turkey; senakal1@,hotmail.com
}

Received 20 July 2013; revised 22 August 2013; accepted 3 September 2013

Copyright (C) 2013 Hakan Öztürk et al. This is an open access article distributed under the Creative Commons Attribution License, which permits unrestricted use, distribution, and reproduction in any medium, provided the original work is properly cited.

\begin{abstract}
Percutaneous nephrolithotomy (PCNL) is very popular and an efficient method as a gold standard in management of renal calculi. It is the first-choice method in management of renal calculi larger than $2 \mathrm{~cm}$. Our patient underwent PCNL upon observing multiple renal calculi larger than $10 \mathrm{~mm}$ in a lower pole of the right kidney. Biopsy was performed during PNCL because morphology and endoscopic view of the calyx were irregular, calcific and pale white in color. The patient developed prolonged urinary leakage from the lumbar region and J-stent was inserted after the re-entry catheter had been removed following successful PCNL. Prolonged urinary leakage persisted although location of the J-stent was normal. Tuberculosis of the urinary tract should be the first option in the differential diagnosis of fistula discharge following PCNL. In our patient, the biopsy taken at the time of PCNL revealed renal tuberculosis. Urinary tract tuberculosis must definitely be considered in the fistula tract persisted and not closed following PCNL as in the present case. Diagnosis of tuberculosis was made early in this case owing to tissue sampling during operation, and thus the treatment was begun early. So we consider this patient as a special case.
\end{abstract}

Keywords: Renal Tuberculosis; PCNL

\section{INTRODUCTION}

Tuberculosis (TB) is a chronic disease caused by $M y$ - cobacterium tuberculosis and Mycobacterium bovis. TB has been a disease threatening the public health since the ancient times. Leading to serious endemics and deaths in the western countries in the $18^{\text {th }}$ and $19^{\text {th }}$ centuries, the disease has begun to decrease independent of chemotherapy since the beginning of the $20^{\text {th }}$ century. In the developing countries, it has begun to spread in the $20^{\text {th }}$ century and the peak point of the endemic has just been reached. Poverty in these countries facing a big endemic, inability to implement tuberculosis control programs, and endemic of HIV/AIDS, especially in the sub-Sahara African and southeast Asian countries, has made it difficult to control the tuberculosis endemic. According to calculations by the World Health Organization (WHO), 8.8 million new cases of TB emerged in 2005 and about 1.6 million people died of tuberculosis [1]. A recent meta-analysis indicated that infection was found in averagely $51.4 \%$ of the persons living in the same house with TBC patients [2]. The persons living in the areas with high incidence of TB, immigrant agricultural workers, the institutionalized persons, HIV carriers, the patients with chronic conditions such as chronic renal, cardiac, pulmonary and hepatic failure, and homeless people are in the high-risk group for tuberculosis [3-5]. Studies carried out in recent years have also indicated that tobacco use significantly increases risk of tuberculosis infection [6]. Estimations from all over the world indicate that almost one third of the global population is infected with tuberculosis. The prevalence of infection is higher in the elderly in the developed countries whereas young adults constitute majority of the cases with infection in the developing countries such as those in Asia, Africa, and Latin America. It has also been shown that the annual risk of disease in tuberculosis-infected patients with HIV varies between $5 \%$ and $15 \%$ depending on the se- 
verity of the immunosuppression [7].

M. tuberculosis, so-called tuberculosis bacillus, is an aerobic, non-sporing, slightly curved or smooth bacillus $0.2-0.6 \times 1-10 \mu \mathrm{m}$ in dimension [8]. The cell wall of the tuberculosis bacillus is much thicker than other bacteria and contains higher amounts of lipids. The main reason for the slow growth rate and drug resistance to many known antibiotics which are distinctive features of the tuberculosis bacillus is low permeability of its cell wall. Rich lipid content as well as less passing canals on the cell wall compared to other bacteria play an important role in low permeability [9]. Diagnosis of the disease is made by microbiological (direct observation, culture), molecular (PCR, strand displacement amplification [SDA], transcription mediated amplification [TMA], ligase chain reaction [LCR]) and immunological (38 $\mathrm{kDa}$ antigen, 16 $\mathrm{kDa}$ antigen, QuantiFERON-TB Gold in tube and $\mathrm{T}$ SPOT-TB) assays [10].

Urinary tuberculosis is a common form of extra-pulmonary tuberculosis. It occurs especially in advanced ages. The disease most commonly starts from the kidneys. The tuberculosis bacillus settles down in the renal cortex during primary bacillemia and forms multiple granulomatous foci. Infection is restricted at this point if immunity is sufficient. Then, some of these lesions are activated and medulla and papilla of the kidney are involved with the advanced infectious process. Desctructive changes occur such as papillary necrosis and cavitation. Typical ulcero-cavernous lesions occur subsequently to ulcerated calyces. At this point, many bacilli and inflammatory debris are discharged into the renal pelvis and then into the ureters and bladder [11-13]. There is no typical clinical sign in urinary tract tuberculosis. The patient may be asymptomatic since the infection develops slowly. Local signs are more common than systemic ones. There is usually a complaint of frequent, painless micturition. Renal tuberculosis rarely causes loss of renal function but previous renal conditions may lead to the development of renal failure. Nephrolithiasis and recurrent bacterial infections may develop in the kidneys $[11,14]$. Diagnosis of genitourinary tuberculosis is made by showing the bacillus in the urine or involved tissue. There are abnormal urinary findings in $90 \%$ of patients with renal or genital tuberculosis. The most common findings are pyuria, hematuria, or combination of them. Tuberculosis should be investigated if pyuria is found in an acidic urine and no microorganism grows in routine urinary culture. In the studies so far, radiological signs related to previous or active tuberculosis on chest radiograms of $50 \%$ to $70 \%$ of patients with genitourinary tuberculosis [12,14]. Culture is positive in $80 \%-95 \%$ of cases of genitourinary tuberculosis. In the study by Bentz et al. [15], association of genitourinary tuberculosis with other forms of extra-pulmonary tuberculosis was found in $21 \%$ of the cases. Genitourinary tuberculosis (GUTB) was demonstrated in $5 \%$ of cases with pulmonary tuberculosis.

While nephrectomy was the mainstay for the management of the renal tuberculosis in the past, it is rarely required currently. Medical treatment is successful in renal tuberculosis and standard anti-tuberculosis treatment has been recommended for 6 months. Monthly urinary culture is also recommended during the treatment until the negative culture is obtained. Nephrectomy is among the therapeutic options in the case of recurrent bacterial infections in the dysfunctional kidney, persisting pain, massive hematuria, hypertension or infection due to multi-drug resistant microorganisms. Surgical or endoscopic interventions may be required to open strictures in the ureters or to increase capacity of the bladder. Nephrostomy or drainage through a stent may be performed in progressive failure develops due to hydronephrosis and obstruction. Use of corticosteroids in addition to a stent in ureterial stenosis is controversial [16]. Genital organ tuberculosis responds well to the anti-tuberculosis treatment. Surgical treatment should be considered only in the presence of pelvic masses not responding to anti-tuberculosis treatment, persisting pelvic pain, and renal tuberculosis advanced to auto-nephrectomy despite medical treatment $[11,12]$.

\section{CASE REPORT}

A 66-year-old man presented to our out-patient clinic with right flank pain and hematuria. The investigations revealed 4 calculi $10 \mathrm{~mm}$ in diameter in the lower pole of right kidney, parenchymal thinning in the lower pole. Both kidneys were functional and both ureters were normal in appearance. His history revealed open surgery for renal calculi from his left kidney, total thyroidectomy, duodenal ulcer perforation, and repair of incisional hernia. His laboratory findings were as follows: Glucose: $102 \mathrm{mg} / \mathrm{dL}$; creatinine: $1.2 \mathrm{mg} / \mathrm{dL}$; hemogram (WBC: 5400/ $\mu \mathrm{L}$; NEU: 57\%; LYM: 30\%; MON: 10\%; Hemoglobin: $12.3 \mathrm{~g} / \mathrm{dL}$; Hematocrit: 35\%, PLT: $320,000 / \mu \mathrm{L}$ ); hepatic function tests: normal; sedimentation rate: $66 /$ hour; TSH: $1.1 \mathrm{uIU} / \mathrm{ml}$; urine analysis: 30 leukocytes, 7 8 erythrocytes per field; Anti-HIV: negative. No bacteria grown in urinary culture. His own and family history didn't reveal tuberculosis.

Pulmonary radiograms were normal. On intravenous pyelography both kidneys were concurrently functional, discontinuity between the right ureter and pelvis, calyceal dilatation in the lower pole of right kidney and 4 renal calculi $10 \mathrm{~mm}$ in diameter were observed. The patient underwent PCNL upon observing multiple renal calculi larger than $10 \mathrm{~mm}$ in lower pole of the right kidney. Biopsy was performed during PNCL because mor- 
phology and endoscopic view of the calyx was irregular, calcific and pale white in color. The patient developed prolonged urinary leakage from lumbar region and $\mathrm{J}$ stent was inserted after re-entry catheter had been removed following successful PCNL. Prolonged urinary leakage persisted although location of the J-stent was normal. Pathological examination of the tissue indicated a few giant cells, histiocytes, and moderately increased lymphocytes, and foci containing caseification necrosis on the regions adjacent to ordinary tubules of the kidney (H \& E, ×4) (Figure 1).

View of giant cell at high magnification $(\mathrm{H} \& \mathrm{E}, \times 40)$, (Figure 2). Acido-resistant staining bacilli in the areas of caseification necrosis (Acido-resistant staining, $\times 100$ ) (Figure 3).

Following consultation with Department of Respiratory Diseases, diagnosis of renal tuberculosis was made and treatment protocol was started with isoniazid, rifampicin, morphozinamide, and ethambutole.

\section{DISCUSSION}

TB has been a disease threatening the public health since the ancient times. The persons living in the areas with high incidence of TB, immigrant agricultural workers, the institutionalized persons, HIV carriers, the patients with chronic conditions such as chronic renal, car-

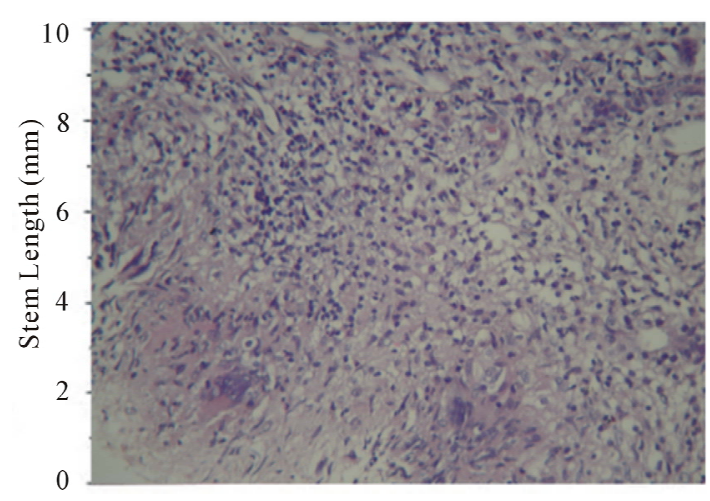

Figure 1. $\mathrm{H} \& \mathrm{E}, \times 4$.

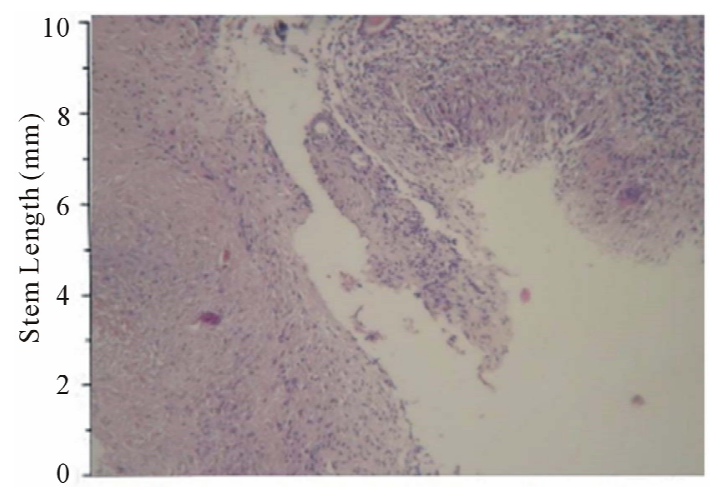

Figure 2. H \& E, $\times 40$.

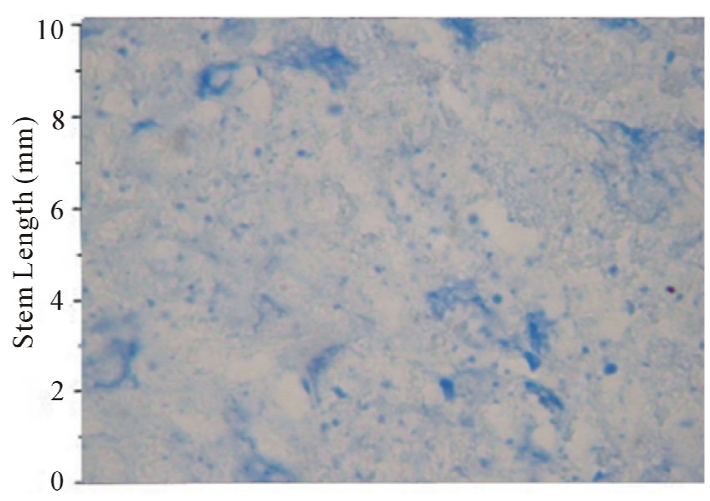

Figure 3. Acido-stant resistaining, $\times 100$.

diac, pulmonary and hepatic failure, and homeless people are in the high-risk group for tuberculosis [3-5]. Genitourinary tuberculosis is a common form of extra-pulmonary tuberculosis. GUTB is a disease with preventable complications if timely diagnosis and treatment are established. In order to avoid outcomes such as renal failure, clinicians need to be aware of signs and symptoms of GUTB when treating urinary problems. Nephrolithiasis and recurrent bacterial infections may develop in the kidneys due to urinary tuberculosis $[11,14]$. The diagnosis of urinary tuberculosis is sometimes difficult, especially when stone disease is present. Both conditions can cause similar radiologic findings and most patients are diagnosed as the urinary stone disease since the radiological findings of this disease are more evident. As in the case presented most of these patients undergo interventions for stone disease and the misdiagnosis of urinary tuberculosis can cause some post-operative difficulties such as urinary fistula formation. Early diagnosis of tuberculosis can prevent these complications. We performed biopsy during PNCL because morphology and endoscopic view of the calyx was irregular, calcific and pale white in color and the diagnosis of tuberculosis was made by pathological examination. This early diagnosis and treatment facilitated the treatment of urinary fistula formation after PCNL. We believe that urinary tuberculosis should be kept in mind in the urinary stone disease patients who live in endemic regions and have a high risk of pulmonary tuberculosis. Urinary tuberculosis can be the main scenario for prolonged urinary fistula after PCNL or other urological interventions in some cases. The urologist should not underestimate this disease because of the increasing incidence of tuberculosis in both developed and under-developed countries.

In conclusion, our patient is asymptomatic and being followed by us with negative tuberculosis-specific urinary culture after quadruple anti-tuberculosis treatment. Tuberculosis of the urinary tract and extra-pulmonary tuberculosis are still a big challenge. Increased the use of immunosuppressive agents, and increased the prevalence 
of several infectious diseases compromising immunity such as HIV, multi-drug resistance, and slowing in discovery of new therapeutic agents have made this problem even more important currently. Because of difficult diagnosis, tuberculosis of urinary tract must be one of the possibilities to be first considered in the case of notclosed urinary fistula following PCNL. The fact that the diagnosis was made by biopsy rather than classical methods and treatment was begun in the early stage was important for our patient in terms of reducing mortality and morbidity.

\section{REFERENCES}

[1] World Health Organization (2009) Global tuberculosis control: Surveillance, planning, financing. WHO report 2009. World Health Organization, Geneva.

[2] Morrison, J., Pai, M. and Hopewell, C.P. (2008) Tuberculosis and latent tuberculosis infection in close contacts of people with pulmonary tuberculosis in low-income and middle-income countries: A systematic review and metaanalysis. The Lancet Infectious Diseases, 8, 359-368. http://dx.doi.org/10.1016/S1473-3099(08)70071-9

[3] Stead, W.W. (1981) Tuberculosis among elderly persons: An outbreak in a nursing home. Annals of Internal Medicine, 94, 606-610. http://dx.doi.org/10.7326/0003-4819-94-5-606

[4] den Boon, S., van Lill, S.W., Borgdorff, M.W., et al. (2005) Association between smoking and tuberculosis infection: A population survey in a high tuberculosis incidence area. Thorax, 60, 555-557. http://dx.doi.org/10.1136/thx.2004.030924

[5] Rieder, H.L. (1999) Epidemiologic basis of tuberculosis. International Union Against Tuberculosis and Lung Disease, Paris.

[6] Bates, M.N., Khalakdina, A., Pai, M., et al. (2007) Risk of tuberculosis from exposure to tobacco smoke. A systematic review and meta-analysis. Archives of Internal Medicine, 167, 335-342.

http://dx.doi.org/10.1001/archinte.167.4.335
[7] Guelar, A., Gatel, J.M., Verdejo, J., et al. (1993) A prospective study of the risk of tuberculosis among HIVinfected patients. AIDS, 7, 1345-1349. http://dx.doi.org/10.1097/00002030-199310000-00007

[8] Barrera, L. (2007) The basics of clinical bacteriology. In: Palomino, J.C., Leao, S.C. and Ritacco, V., Eds., Tuberculosis 2007: From Basic Science to Patient Care, Sao Paulo, 93-112. www.TuberculosisTextbook.com

[9] Niederweis, M. (2003) Mycobacterial porins: New channel proteins in unique outer membranes. Molecular Microbiology, 49, 1167-1177. http://dx.doi.org/10.1046/j.1365-2958.2003.03662.x

[10] Goletti, D., Stefania, C., Butera, O., et al. (2008) Accuracy of immunodiagnostic tests for active tuberculosis using single and combined results: A multicenter TBNETstudy. PLOS Medicine, 3, Article ID: e3417.

[11] Iseman, M.D. (2000) A clinician's guide to tuberculosis. Lippincot, Williams, Philadelphia.

[12] Hopewell, P.C. (2005) Tuberculosis and other mycobacterial diseases. In: Mason, R.J., Murray, J.F., Broaddus, V.C. and Nadel, J.A., Eds., Murray and Nadel's Textbook of Respiratory Medicine, 4th Edition, Elsevier Saunders, Philadelphia, 979-1043.

[13] Golden, M.P. and Vikram, H.R. (2005) Extrapulmonary tuberculosis: An overview. American Family Physician, 72, 1761-1768.

[14] Gow, J.G. (1999) Genitourinary tuberculosis In: Schlossberg, D., Ed., Tuberculosis and Nontuberculous Mycobacterial Infections, 4th Edition, Saunders, Philadelphia, 195-203.

[15] Bentz, R.R., Dimcheff, D.G., Nemeroff, M.J., et al. (1975) The incidence of urine cultures positive for M. Tuberculosis in a general tuberculosis patient population. American Review of Respiratory Disease, 111, 647-650.

[16] American Thoracic Society/Centers for Disease Control and Prevention/Infectious Diseases Society of America (2005) A controlling tuberculosis in the United States. American Journal of Respiratory and Critical Care Medicine, 172, 1169-1227. http://dx.doi.org/10.1164/rccm.2508001 